\title{
Family meals to fast food: findings from a systematic review of childhood and adolescent cohorts which measure whole diet and subsequent adiposity
}

\author{
C.E. Rycroft, C.E.L. Evans and J.E. Cade \\ Nutritional Epidemiology Group, School of Food Science and Nutrition, University of Leeds, Leeds, LS2 9JT
}

In 2013 around $23 \%$ of children living in developed countries and approximately $13 \%$ of children living in the developing world were overweight or obese ${ }^{(1)}$. Intervention studies show promising obesity prevention strategies ${ }^{(2)}$ but there are gaps in the published research about childhood diet and associated obesity risk. Small associations between dietary patterns and adverse changes in fat mass during mid-childhood, found in European cohorts, show that longitudinal studies may be a useful way to investigate dietary risk factors for future obesity. This systematic review identified childhood and adolescent cohorts with a minimum follow up of 2 years to address the research question: to what extent does diet during childhood and adolescence influence future indicators of overweight or obesity?

The PRISMA_P 2015 statement $^{(3)}$ guided protocol development. Search terms, generated using a PICO (Patients, Interventions, Comparators, Outcomes) framework, were (Children or Adolescents) and (Diet or Nutrition or Food intake or Diet quality) and (Body composition or Height and Weight). Ovid Medline, Embase, Cochrane CENTRAL, Web of Science and Scopus were searched for articles published from January 1990, with no limit on language. Full text articles in English were included if they were from a cohort study where over half the participants were healthy children/adolescents aged 8 to 19 years old at baseline AND whole diet was measured AND adiposity outcomes were measured at least 2 years after diet AND an association between a dietary exposure and an adiposity outcome was reported. Internal validity of included records was assessed using a modified version of the Newcastle-Ottawa quality assessment tool for cohort studies. Data was extracted into Excel and into STATA for meta-analysis if appropriate.

Literature searches found 11,604 records. After removing duplicates and irrelevant titles, two independent reviewers screened 3,431 abstracts and then 390 full texts. After third review and quality assessment 35 papers from 14 observational studies were included. Nine cohorts, including two girls only cohorts, were from U.S.A, three from Europe, one from South America and one from Australia. The largest cohort recruited 14,000+ participants. Sample sizes in analyses ranged from 100+ to 6,500 children, aged 3 to 15 years old at first measure. Studies explored the intake of foods/drinks, eating habits, dietary patterns and diet quality and adiposity outcomes including change in body mass index, BMIz score, waist circumference or body fat percentage. Few studies used similar measures, making comparison difficult. Reported associations included family meals which were predictive of lower adiposity measures, whereas fast foods (fried food away from home) and energy dense dietary patterns were associated with increased adiposity. However in analyses that adjusted for total energy intake, reported associations were often attenuated or no longer significant. Opportunities for formal quantitative synthesis by meta-analysis were limited.

High quality childhood/adolescent cohorts which measure whole diet and later adiposity do exist but they are under exploited. Use of standardised measurement and reporting protocols could increase their potential to investigate childhood/adolescent dietary risk factors for future obesity.

1. Ng M, Fleming T, Robinson M et al. (2013) Lancet 384 (9945):766-81.

2. Waters E, de Silva-Sanigorski A, Hall BJ et al. (2011) Cochrane Db Syst Rev (12): CD001871.

3. Moher D, Shamseer L, Clarke M et al. (2015) Syst Rev 4(1). 